\title{
How not to approach coronary bifurcation
}

\section{Nikola Bakracheski*, Elena Kovacheska, Dejan Mancheski, Deni Razmoski, Jasmina Spaseska}

Institute of Cardiovascular Diseases, Ohrid, Macedonia
KEYWORDS: bifurcational, stenting, percutaneous coronary intervention. CITATION: Cardiol Croat. 2018;13(11-12):389. | https://doi.org/10.15836/ccar2018.389 *ADDRESS FOR CORRESPONDENCE: Nikola Bakracheski, 6000 Ohrid, Macedonia. / Phone: +389-76-225-500 E-mail: nbakrac@yahoo.com

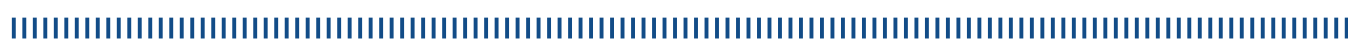

Background: Almost all described and well established bifurcational stenting techniques need to be performed with $6 \mathrm{~F}$ coronary guide catheters at least. ${ }^{1}$

Methods: 62-years-old female patient was admitted in our hospital for elective coronary angiography due to effort angina and positive coronary stress test. Due to radial artery spasm, $5 \mathrm{~F}$ diagnostic catheters were used to perform the coronary angiogram and it revealed Medina 0,0,1 bifurcational lesion of left anterior descending artery/diagonal branch 1 (LAD/Dgl) There was no significant stenosis on right coronary artery (RCA). We approached percutaneous coronary intervention (PCI) of the ostium of Dgl using 5F extra-backup (EBU) 3.5 guide catheter (GC) and Ballance Middle Weight (BMW) guidewire in the Dgl, while LAD wasn't wired. Predilatation was made with 2.0x20 mm semi-compliant (SC) balloon catheter and 2.25x18 mm Drug-eluting stent (DES) was deployed covering the ostium of the Dgl and $2 \mathrm{~mm}$ proximal to the LAD. Control angiography showed significant residual stenosis of the distal part of the main branch (LAD) due to plaque and carina shift. We successfully exchanged 5F EBU 3.5 with 6F EBU 3.5 guide catheter after the intra-arterial application of nitroglycerin in the radial artery and continued PCI using modified mini-crush bifurcational technique. Main branch was wired and side branch stent was crushed with 2.75x15 mm SC balloon catheter. After side branch wire removal, 3.0x20 DES was deployed in LAD and side branch was rewired. Final kissing balloon inflation with 2.75x15 $\mathrm{mm}$ and 2.25x15 $\mathrm{mm}$ noncompliant (NC) balloons was performed in the end.

Results: After compromising the main branch vessel with the side branch stent deployment, we promptly changed our previously intended one-stent strategy to two-stent strategy using modified mini-crush technique, which was facilitated with guiding catheter exchange from 5F to $6 \mathrm{~F} \mathrm{GC}$ and wiring the both vessels. Changing the plan during the procedure resulted in prolonged procedural time (additional $18 \mathrm{~min}$ ), increased total radiation dose and amount of the contrast media used (70 $\mathrm{ml}$ more). The procedure underwent without serious complications with satisfactory final result, utilizing mini-crush technique without residual stenosis of both vessels.

Conclusion: Mandatory strategy planning and appropriate materials selection before approaching any bifurcational coronary lesion are the essence of every safely-performed and efficient PCI.
RECEIVED:

October 25, 2018

ACCEPTED:

November 5, 2018

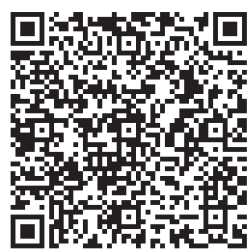

LITERATURE IIIIIIIIIIIIIIIIIIIIIIIIIIIIIIIIIIIIIIIIIIIIIIIIIIIIIIIIIIIIIIIIIIIIIIIIIIIIIIIIIIIIIIIIIIIIIIIIIIIIIII

1. Sawaya FJ, Lefèvre T, Chevalier B, Garot P, Hovasse T, Morice MC, et al. Contemporary Approach to Coronary Bifurcation Lesion Treatment. JACC Cardiovasc Interv. 2016 Sep 26;9(18):1861-78. https://doi.org/10.1016/j.jcin.2016.06.056 